cambridge.org/neu

\section{Short Communication}

Cite this article: Chen S, Mizoue $\mathrm{T}, \mathrm{Hu} \mathrm{H}$, Kuwahara K, Honda T, Yamamoto S, Nakagawa T, Miyamoto T, Okazaki H, Shimizu M, Murakami T, Eguchi M, Kochi T, Yamamoto M, Ogasawara T, Sasaki N, Uehara A, Imai T, Nishihara A, Hori A, Nagahama S, Tomita K, Konishi M, Kabe I, Dohi S, and for the Japan Epidemiology Collaboration on Occupational Health Study Group. (2019) Serum cholesterol levels preceding to suicide death in Japanese workers: a nested casecontrol study. Acta Neuropsychiatrica 31: 266-269. doi: 10.1017/neu.2019.26

Received: 6 February 2019

Revised: 27 May 2019

Accepted: 3 June 2019

First published online: 1 July 2019

\section{Key words:}

suicide; cholesterol; nested case-control study; working population

\section{Author for correspondence:}

Sanmei Chen, Email: schen@hosp.ncgm.go.jp

\title{
Serum cholesterol levels preceding to suicide death in Japanese workers: a nested case-control study
}

\author{
Sanmei Chen ${ }^{1}\left(\mathbb{D}\right.$, Tetsuya Mizoue ${ }^{1}$, Huanhuan $\mathrm{Hu}^{1}$, Keisuke Kuwahara ${ }^{1,2}$, \\ Toru Honda ${ }^{3}$, Shuichiro Yamamoto ${ }^{3}$, Tohru Nakagawa ${ }^{3}$, Toshiaki Miyamoto ${ }^{4}$, \\ Hiroko Okazaki ${ }^{5}$, Makiko Shimizu ${ }^{6}$, Taizo Murakami ${ }^{6}$, Masafumi Eguchi ${ }^{7}$, \\ Takeshi Kochi ${ }^{7}$, Makoto Yamamoto ${ }^{8}$, Takayuki Ogasawara ${ }^{9}$, Naoko Sasaki ${ }^{9}$, \\ Akihiko Uehara $^{10}$, Teppei Imai ${ }^{11}$, Akiko Nishihara ${ }^{12}$, Ai Hori ${ }^{13}$, Satsue Nagahama ${ }^{14}$, \\ Kentaro Tomita ${ }^{1}$, Maki Konishi ${ }^{1}$, Isamu Kabe ${ }^{7}$, Seitaro Dohi ${ }^{5}$ and for the Japan \\ Epidemiology Collaboration on Occupational Health Study Group
}

${ }^{1}$ Department of Epidemiology and Prevention, National Center for Global Health and Medicine, Tokyo, Japan ${ }^{2}$ Teikyo University Graduate School of Public Health, Tokyo, Japan; ${ }^{3}$ Hitachi, Ltd., Ibaraki, Japan; ${ }^{4}$ Nippon Steel \& Sumitomo Metal Corporation Kimitsu Works, Chiba, Japan; ${ }^{5}$ Mitsui Chemicals, Inc., Tokyo, Japan; ${ }^{6}$ Mizue Medical Clinic, Keihin Occupational Health Center, Kanagawa, Japan; ${ }^{7}$ Furukawa Electric Co., Ltd., Tokyo, Japan; ${ }^{8}$ Yamaha Corporation, Shizuoka, Japan; ${ }^{9}$ Mitsubishi Fuso Truck and Bus Corporation, Kanagawa, Japan; ${ }^{10}$ Seijinkai Shizunai Hospital, Hokkaidō, Japan; ${ }^{11}$ Azbil Corporation, Tokyo, Japan; ${ }^{12}$ Department of Global Public Health, University of Tsukuba, Ibaraki, Japan; ${ }^{13}$ All Japan Labour Welfare Foundation, Tokyo, Japan and ${ }^{14}$ Mitsubishi Plastics, Inc., Tokyo, Japan

\section{Abstract}

Objective: To investigate the association between suicide death and serum cholesterol levels as measured at times close to suicide death. Methods: We conducted a nested case-control study of 41 cases of suicide deaths and 205 matched controls with serum total cholesterol (TC) levels till 3 years before suicide death in a large cohort of Japanese workers. Results: Individuals in the lowest versus highest tertile/predefined category of TC in a Japanese working population had a three- to four-fold greater risk of suicide death. Each $10 \mathrm{mg} / \mathrm{dl}$ decrement of average TC was associated with an $18 \%$ increased chance of suicide death (95\% confidence interval, $2-35 \%$ ). Similar results were found for TC levels at each year. Conclusion: These results suggest that a low serum TC level in recent past is associated with an increased risk of suicide death.

\section{Significant outcomes}

- A lower averaged level of TC across 3 years before suicide death was significantly associated with an increased risk of suicide death.

- This association remained statistically significant when the averaged TC level was treated as a continuous or a categorical variable.

- Similar results were found for TC levels at each single year.

\section{Limitations}

- A relatively small number of cases.

- The possibility of bias due to residual confounding effects and unmeasured confounders.

- The limited generalisability of the results to populations with different backgrounds.

\section{Introduction}

Suicide is among the leading causes of death worldwide. In Japan, which has one of the highest global suicide rates, suicide is the top cause of death among young adults (Ministry of Health Labour and Welfare of Japan, 2018). Suicide, in addition to several psychiatric disorders (De Berardis et al., 2012, 2017, 2014), has been linked to nutritional biomarkers including serum cholesterol (Mittendorfer \& Wasserman, 2000). Low serum total cholesterol (TC) level has been found to be related to lower central nervous system serotonin activity (Steegmans et al., 1996), a condition often observed in cases of depression and suicide. However, epidemiological evidence regarding the association between serum TC concentrations and the risk of suicide death is 
conflicting, with some studies showing an inverse association (Lindberg et al., 1992; Neaton et al., 1992; Zureik et al., 1996; Partonen et al., 1999; Ellison \& Morrison, 2001; Jee et al., 2011) and others suggesting a null (Kromhout et al., 1992; Smith et al., 1992; Giltay et al., 2010; Chang et al., 2012) or even positive (Iribarren et al., 1995; Tanskanen et al., 2000; Svensson et al., 2017) association. Furthermore, no data are available regarding circulating TC levels during the years immediately preceding suicide death. In this study, we examined serum TC levels as measured during the 3 years before suicide death among a large cohort of Japanese workers. We hypothesised that low levels of serum TC are associated with an increased risk of suicide death.

\section{Materials and methods}

\section{Study design}

We performed a case-control study nested in a cohort of the Japan Epidemiology Collaboration on Occupational Health (J-ECOH) Study, which is an ongoing study among workers at multiple companies (Hu et al., 2018). A total of 146619 participants who attended health check-ups at least once between January 2008 and December 2016 or between April 2008 and March 2017 comprised the sample for the present study. The research protocol was approved by the Ethics Committee of the National Center for Global Health and Medicine in Japan.

\section{Measures}

\section{Ascertainment of suicide death and control selection}

Using within-study registration, we ascertained participants' deaths and their causes of death since April 2012. The dataset was locked for the present study on 10 August 2018. Causes of deaths were confirmed based on death certificates, sick leave documents, family confirmation and other sources. The causes of deaths were classified according to the International Statistical Classification of Diseases and Related Health Problems, 10th revision (ICD-10). Of the 60 suicide deaths (ICD-10 codes X60-X84) we ascertained, 19 cases were excluded owing to a lack of TC measurements conducted at any health check-up during the 3 years prior to suicide death, leaving 41 cases for analysis. Controls were selected by using the incidence density method. For each case of suicide death, we first created a pool of controls matched on sex, birthday ( \pm 2 years) and worksite. We then allocated the date of suicide death as an index date to matched controls. For a given case, we randomly selected five controls from the pool of eligible controls. We did not allow cases to be chosen as controls, nor did we allow a selected control to serve as a control of other cases.

\section{Serum total cholesterol and other variables}

Serum TC levels were analysed enzymatically. Analyses of TC were performed by laboratories that hold a high rank (Rank A or Score > 95/100 according to external quality control agencies). Of 41 cases, 28 had three recorded TC measurements, 5 had two TC records, and 8 had only one TC measurement. We calculated a mean TC level using the available data. Covariates included smoking status, lipid-lowering treatment, diabetes and hypertension at 1 year prior to suicide death. If these data were not available, measurements obtained 2 or 3 years prior to death were used.

\section{Statistical analyses}

We built conditional logistic regression models to estimate odds ratios (OR) and 95\% confidence intervals (CI) of suicide death according to the 3 -year average of TC levels. We used continuous (each $10 \mathrm{mg} / \mathrm{dl}$ decrement), tertile and predefined categories of averaged TC ( $<180 \mathrm{mg} / \mathrm{dl}, 180$ to $<200 \mathrm{mg} / \mathrm{dl}, 200$ to $<220$ $\mathrm{mg} / \mathrm{dl}$ and $\geq 220 \mathrm{mg} / \mathrm{dl}$ ) as independent variables in separate models. We also adjusted for current smoking status, lipid-lowering treatment, diabetes and hypertension. We assessed trend association by assigning ordinal numbers to TC categories and modelling this as a continuous variable. We repeated the analyses using TC levels at single time points. We tested the interaction of smoking status on the potential association between TC and the risk of suicide death. We performed two sensitivity analyses: (1) excluding subjects who were undergoing lipid-lowering treatment and (2) using data comprising cases that had two or more TC measurements and their matched controls. Statistical analyses were performed using SAS version 9.4.

\section{Results}

There were no significant differences in the mean of body mass index, glycated hemoglobin, systolic blood pressure and diastolic blood pressure, nor in the rate of current smoker, lipid-lowering treatment, diabetes or hypertension between cases and controls (Table 1). Cases had significantly higher blood glucose levels than did controls.

The risk of suicide death increased with decreasing serum TC levels across the 3 years preceding suicide death (Table 2). Compared with the highest tertile $(\geq 217 \mathrm{mg} / \mathrm{dl})$, the adjusted OR $(95 \%$ CI) was $1.49(0.53,4.16)$ for the middle tertile and $4.68(1.67,13.14)$ for the lowest tertile $(<188 \mathrm{mg} / \mathrm{dl})$ ( $p$ for trend $=0.003$ ). In analyses using the predefined categories with TC levels $\geq 220 \mathrm{mg} / \mathrm{dl}$ as a reference, the adjusted OR $(95 \%$ CI) was $1.72(0.53,5.55)$ for TC levels 200 to $<220 \mathrm{mg} / \mathrm{dl}, 2.80$ $(0.96,8.14)$ for TC levels 180 to $<200 \mathrm{mg} / \mathrm{dl}$ and $3.29(1.10,9.85)$ for TC levels $<180 \mathrm{mg} / \mathrm{dl}$ ( $p$ for trend =0.02). Each $10 \mathrm{mg} / \mathrm{dl}$ decrement of average TC was associated with an $18 \%$ increased chance of suicide death (95\% CI, 2-35\%). The interaction between smoking status and average TC was not statistically significant ( $p$ for interaction $=0.17$ ). The results were similar when examining the associations using TC levels recorded during each year before suicide/index date (Supplementary Table 1).

In sensitivity analyses, the exclusion of cases and controls who were undergoing lipid-lowering treatment did not materially change the results (Supplementary Table 2). Similar results were obtained when restricting analyses to cases with two or more TC measurements and their matched controls (data not shown).

\section{Discussion}

In the present case-control study nested in a large cohort study of a Japanese working population, we demonstrated that low serum TC levels during the years immediately preceding suicide were significantly associated with an increased risk of suicide death. To our knowledge, this is the first study to demonstrate this association using serum TC levels measured at time points close to suicide death.

A meta-analysis of seven Western cohort studies showed a twofold increased risk of suicide death for the lowest versus highest TC (Wu et al., 2016). In our study, individuals in the lowest versus 
Table 1. Characteristics of suicide cases and controls at 1 year prior to suicide/index date

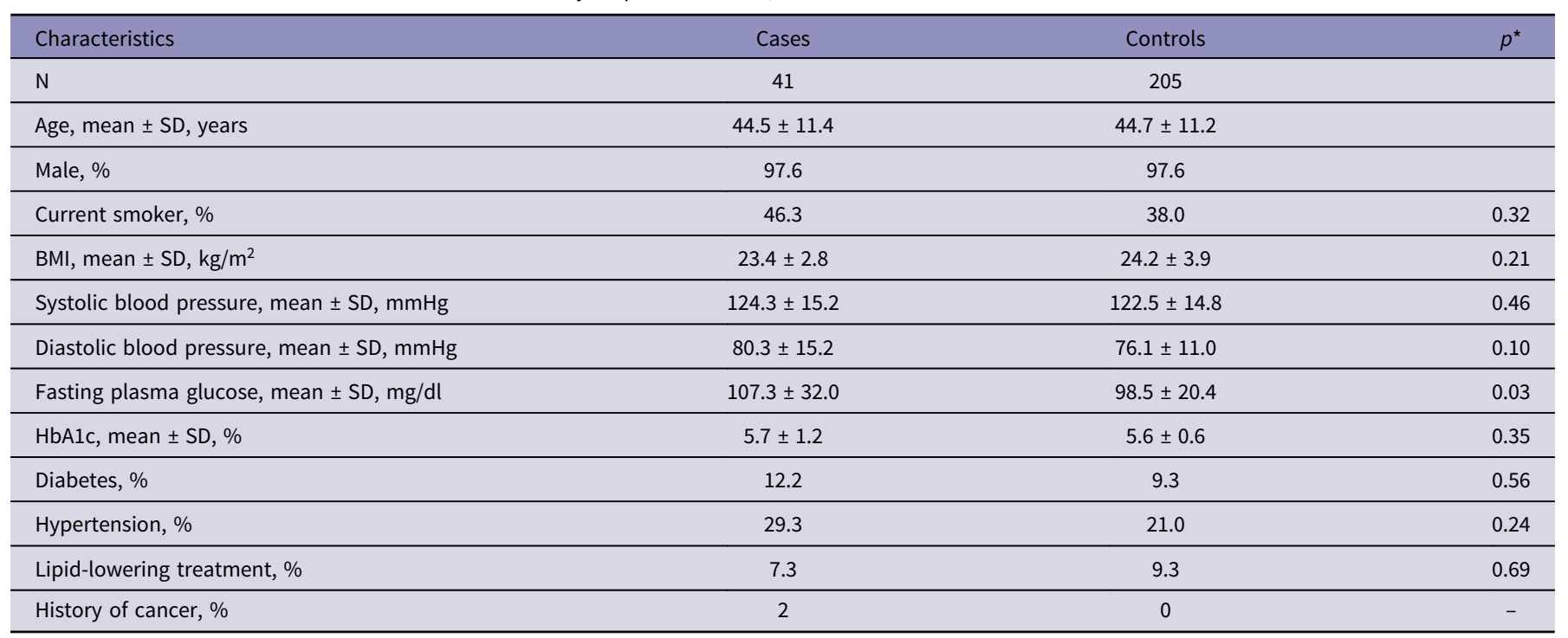

SD, standard deviation; BMI, body mass index; HbAlc, glycated hemoglobin.

${ }^{\star}$ Comparisons between cases and controls using chi-square test for categorical variables, $t$-test for continuous variables.

Table 2. Associations between 3-year average of serum total cholesterol (TC) levels and the risk of suicide death

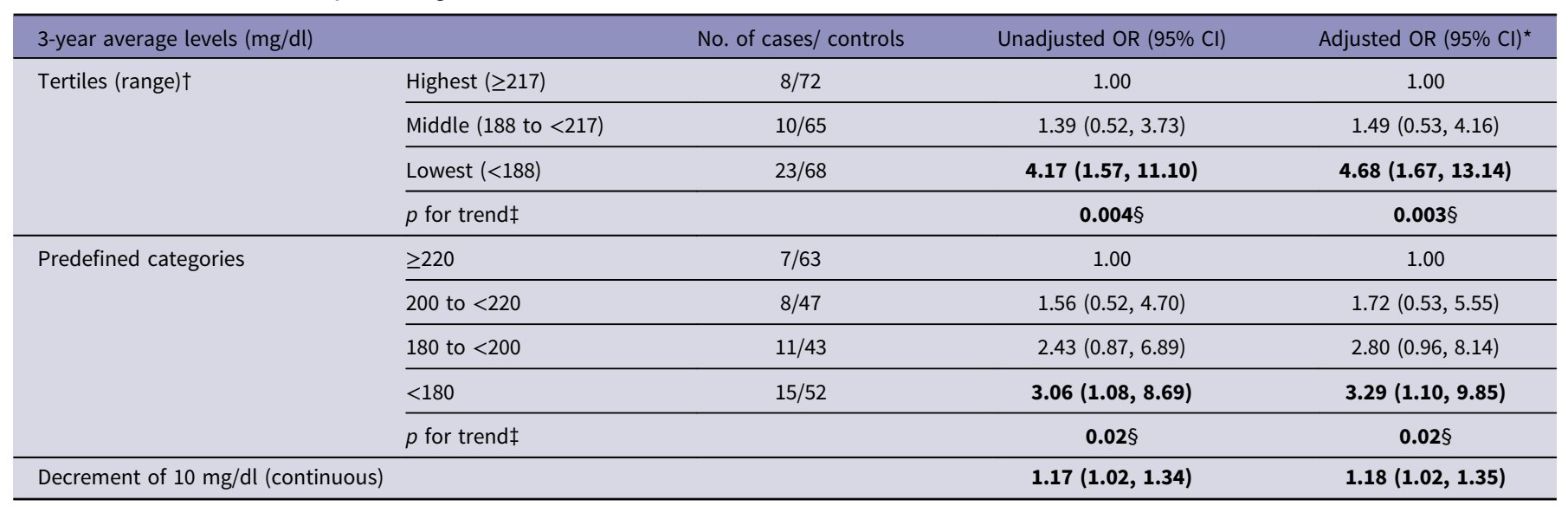

OR, odds ratio; $\mathrm{Cl}$, confidence interval.

*Adjusted for smoking (current smoker or not), lipid-lowering treatment (yes or no), diabetes (yes or no) and hypertension (yes or no).

†Tertiles were based on the distribution of TC levels among controls.

$\ddagger$ Trend association was assessed by assigning ordinal numbers to each tertile and treating this variable as a continuous variable.

$\S p<0.05$.

highest tertile/predefined category had a three- to four-fold higher risk of suicide death. Our estimates were within the range of relative risks demonstrated in previous prospective studies showing an inverse association between TC and suicide death (approximately two- to six-fold higher risk for the lowest versus highest quartile/predefined category) (Lindberg et al., 1992; Neaton et al., 1992; Zureik et al., 1996; Partonen et al., 1999; Ellison \& Morrison, 2001; Jee et al., 2011). The present study, conducted in a Japanese working population, not only confirms previous findings among primarily Western populations but also provides additional evidence to link suicide risk to TC levels in the recent past.

A Finnish study reported a twofold increased risk of suicide death associated with a TC level $\geq 309 \mathrm{mg} / \mathrm{dl}$ (vs. $<193 \mathrm{mg} / \mathrm{dl}$ ) (Tanskanen et al., 2000). A Japanese study showed an approximately twofold increased risk of suicide death associated with a TC level $\geq 220 \mathrm{mg} / \mathrm{dl}$ (vs. 180 to $<220 \mathrm{mg} / \mathrm{dl}$ ) in women but not in men (Svensson et al., 2017). In the present study, owing to the presence of few cases with a TC level of $\geq 240 \mathrm{mg} / \mathrm{dl} \quad(n=4)$, we were unable to assess suicide risk associated with high TC levels.

The strengths of this study are its repeated measurements of serum TC levels and the prospective design in a well-defined cohort study. However, there are also some limitations to consider. First, the number of cases $(n=41)$ was not large relative to that of previous studies. Despite this, we observed a statistically significant association between serum TC and the risk of suicide death. Second, we cannot rule out the possibility of bias due to residual confounding effects and unmeasured confounders, such as job stress. Third, as participants were employees at large companies in a Japanese context, we urge caution in generalising the results to populations with different backgrounds.

In conclusion, our findings among a Japanese working population add evidence to support the hypothesis that low serum TC level in the 
recent past is associated with an increased risk of suicide death. The potential of monitoring serum TC level as a target metric for suicide prevention interventions needs to be further explored.

Supplementary material. To view supplementary material for this article, please visit https://doi.org/10.1017/neu.2019.26.

\section{Author ORCIDs. Chen Sanmei (DD 0000-0003-0811-1701}

Acknowledgements. We thank Dr. Toshiteru Okubo (Chairperson of Industrial Health Foundation) for scientific advice on the conduct of J-ECOH Study and Ms. Rika Osawa (National Center for Global Health and Medicine) for administrative supports.

Author contributions. All authors made substantive contributions to the study. SC and TM designed the study. TM and SD designed the J-ECOH project. SC, TM, HH and KK contributed to the statistical analyses. SC, TM, HH, KK, TH, SY, TN, TM, HO, MS, TM, ME, TK, MY, TO, NS, AU, TI, AN, AH, SN, KT, MK, IK and SD collected data and contributed to the interpretations of the data. SC, TM, HH and KK drafted the manuscript. All authors contributed to the revision of the draft. All authors have read and given final approval of the latest version of the manuscript.

Financial support. This study was supported by the Industrial Health Foundation, Industrial Disease Clinical Research Grants (140202-01, 150903-01, 170301-01), Japan Society for the Promotion of Science KAKENHI (16H05251), Japan Society for the Promotion of Science Grant-in-Aid for Young Scientists (19K19474); and Grant of National Center for Global Health and Medicine (28-Shi-1206). The funding sources had no role in the study design, collection, analyses and interpretation of the data, writing of the report or the decision to submit the paper for publication.

Conflict of interest. We declared no conflicts of interest relevant to this article.

\section{References}

Chang S, Wen CP, Tsai MK, Lawlor DA, Yang YC and Gunnell D (2012) Adiposity, its related biologic risk factors, and suicide: a cohort study of 542,088 Taiwanese adults. The American Journal of Epidemiology 175, 804-815.

De Berardis D, Marini S, Piersanti M, Cavuto M, Perna G, Valchera A, Mazza M, Fornaro M, Iasevoli F, Martinotti G and Di Giannantonio M (2012) The relationships between cholesterol and suicide: an update. ISRN Psychiatry 2012, 1-6.

De Berardis D, Serroni N, Campanella D, Marini S, Rapini G, Valchera A, Iasevoli F, Mazza M, Fornaro M, Perna G, Di Iorio G, Martinotti G and Di Giannantonio M (2017) Alexithymia, suicide ideation, C-reactive protein, and serum lipid levels among outpatients with generalized anxiety disorder. Archives of Suicide Research 21, 100-112.

De Berardis D, Serroni N, Marini S, Rapini G, Carano A, Valchera A, Iasevoli F, Mazza M, Signorelli M, Aguglia E, Perna G, Martinotti G, Varasano PA, Pressanti GL and Di Giannantonio M (2014) Alexithymia, suicidal ideation, and serum lipid levels among drug-nä̈ve outpatients with obsessive-compulsive disorder. Revista Brasileira de Psiquiatria $36,125-130$.

Ellison LF and Morrison HI (2001) Low serum cholesterol concentration and risk of suicide. Epidemiology 12, 168-172.
Giltay EJ, Zitman FG, Menotti A, Nissinen A, Jacobs DRJr., Adachi H, Kafatos A, Kromhout D, Seven Countries Study Group (2010) Respiratory function and other biological risk factors for completed suicide: 40 years of follow-up of European cohorts of the Seven Countries Study. The Journal of Affective Disorders 120, 249-253.

Hu H, Mizoue T, Sasaki N, Ogasawara T, Tomita K, Nagahama S, Hori A, Nishihara A, Imai T, Yamamoto M, Eguchi M, Kochi T, Miyamoto T, Honda T, Nakagawa T, Yamamoto S, Okazaki H, Uehara A, Shimizu M, Murakami T, Kuwahara K, Nanri A, Konishi M, Kabe I, Dohi S, Japan Epidemiology Collaboration on Occupational Health Study Group (2018) Prediabetes and cardiovascular disease risk: a nested case-control study. Atherosclerosis 278, 1-6.

Iribarren C, Reed DM, Wergowske G, Burchfiel CM and Dwyer JH (1995) Serum cholesterol level and mortality due to suicide and trauma in the Honolulu Heart Program. Archives of Internal Medicine 155, 695-700.

Jee SH, Kivimaki M, Kang H-C, Park IS, Samet JM and Batty GD (2011) Cardiovascular disease risk factors in relation to suicide mortality in Asia: prospective cohort study of over one million Korean men and women. European Heart Journal 32, 2773-2780.

Kromhout D, Katan M, Menotti A, Keys A and Bloemberg B (1992) Serum cholesterol and long-term death rates from suicide, accidents, or violence. Lancet 340, 317-318.

Lindberg G, Råstam L, Gullberg B and Eklund GA (1992) Low serum cholesterol concentration and short term mortality from injuries in men and women. British Medical Journal 305, 277-279.

Ministry of Health Labour and Welfare of Japan (2018) National Vital Statistics. Available at https://www.mhlw.go.jp/toukei/saikin/hw/jinkou/ kakutei17/index.html (accessed 29 November 2018).

Mittendorfer E and Wasserman D (2000) Literature Review: Relationship Between Cholesterol and Suicide. Stockholm, Sweden: National Centre for Suicide Research and Prevention of Mental Ill-health.

Neaton JD, Blackburn H, Jacobs D, Kuller L, Lee DJ, Sherwin R, Shih J, Stamler J and Wentworth D (1992) Serum cholesterol level and screened in the Multiple Risk Factor Intervention Trial Group. Archives of Internal Medicine 152, 1490-1500.

Partonen T, Haukka J, Virtamo J, Taylor PR and Lonnqvist J (1999) Association of low serum total cholesterol with major depression and suicide. British Journal of Psychiatry 175, 239-262.

Smith GD, Shipley MJ, Marmot MG and Rose G (1992) Plasma cholesterol concentration and mortality. The Journal of the American Medical Association 267, 70-76.

Steegmans PHA, Fekkes D, Hoes AW, Bak AAA, van der Does E and Grobbee DE (1996) Low serum cholesterol concentration and serotonin metabolism in men. British Medical Journal 312, 221.

Svensson T, Inoue M, Sawada N, Charvat $\mathrm{H}$, Mimura $\mathrm{M}$ and Tsugane $\mathrm{S}$ (2017) High serum total cholesterol is associated with suicide mortality in Japanese women. Acta Psychiatrica Scandinavica 136, 259-268.

Tanskanen A, Vartiainen E, Tuomilehto J, Viinamäki H, Lehtonen J and Puska P (2000) High serum cholesterol and risk of suicide. The American Journal of Psychiatry 157, 648-650.

Wu S, Ding Y, Wu F, Xie G, Hou J and Mao P (2016) Serum lipid levels and suicidality: a meta-analysis of 65 epidemiological studies. The Journal of Psychiatry \& Neuroscience 41, 56-69.

Zureik M, Courbon D and Ducimetière P (1996) Serum cholesterol concentration and death from suicide in men: Paris prospective study I. British Medical Journal 313, 649-651. 